\title{
COMPARAÇÃO DO CONSUMO ALIMENTAR CALCULADO A PARTIR DE DUAS TABELAS DE COMPOSIÇÃO DE ALIMENTOS
}

\section{Camila Tamires de Castilhos Romani}

Nutricionista pela Pontifícia Universidade Católica do Rio Grande do Sul (PUCRS). Brasil.

\section{Evelyn Carvalho de Lima}

Nutricionista pela Pontifícia Universidade Católica do Rio Grande do Sul (PUCRS). Brasil.

\section{Vera Elizabeth Closs}

Doutora em Gerontologia Biomédica pela Pontifícia Universidade Católica do Rio Grande do Sul (PUCRS), Porto Alegre (RS), Brasil.

\section{Fabrício Edler Macagnan}

Docente adjunto da Universidade Federal de Ciências da Saúde de Porto Alegre (UFCSPA), Docente e Tutor no Programa de Residência Multiprofissional Integrada em Saúde com ênfase em Onco-Hematologia desenvolvido em conjunto com a Irmandade Santa Casa de Misericórdia de Porto Alegre (ISCMPA) e Docente no Programa de Pós-Graduação em Ciências da Reabilitação (UFCSPA), Brasil

\section{Margareth da Silva Oliveira}

Doutorado em Psiquiatria e Psicologia Médica pela Universidade Federal de São Paulo e Pós-Doutorado na University of Maryland Baltimore County (UMBC-USA). Pesquisadora Produtividade CNPq-1C, Docente titular da Pontifícia Universidade Católica do Rio Grande do Sul (PUCRS), Brasil.

\section{Andréia da Silva Gustavo}

Doutorado em Epidemiologia pela Universidade Federal do Rio Grande do Sul (UFRGS). Professora adjunta e Coordenadora do Curso de Graduação em Enfermagem da Pontifícia Universidade Católica do Rio Grande do Sul (PUCRS), Brasil.

\section{Ana Maria Pandolfo Feoli}

Doutorado em Ciências Biológicas (Bioquímica) pela Universidade Federal do Rio Grande do Sul (UFRGS). Docente e pesquisadora do Curso de Nutrição e do Pós Graduação em Psicologia da Pontifícia Universidade Católica do Rio Grande do Sul (PUCRS), Brasil.
RESUMO: O objetivo do presente trabalho foi comparar o consumo de energia, macronutrientes e micronutrientes calculado a partir de duas tabelas de composição dos alimentos: Tabela Brasileira de Composição de Alimentos (TACO) mais informações de rótulos de alimentos e United States Department of Agriculture Food Composition Databases (USDA). Trata-se de estudo transversal, com dados do consumo alimentar de participantes do estudo principal "Modificação do Estilo de Vida e Risco Cardiovascular". Os dados foram analisados por meio dos Testes de Kruskal Wallis, Post Hoc de Dunn, e Correlação de Pearson. Foram avaliados 59 Recordatórios de 24 horas e as medianas de 21 entre 29 nutrientes $(72,4 \%)$ apresentaram diferença estatisticamente significativa $(p<0,05)$ na comparação entre os resultados obtidos das tabelas avaliadas. Foram encontradas divergências significativas entre os valores nutricionais apresentados nas tabelas. Este fato alerta para o uso destes instrumentos e a sua influência nos resultados do cálculo do consumo alimentar e impacto na prescrição nutricional.

PALAVRAS-CHAVE: Análise de Alimentos; Consumo de Alimentos; Nutrientes; Tabela de Composição dos Alimentos.

\section{COMPARING FOOD INTAKE CALCULATED BY T WO LISTS OF FOOD COMPOSITION}

ABSTRACT: Energy, macronutrient and micronutrient intakes are
compared by two food composition lists: Brazilian Food Composition
List and nutrition facts and the United States Department of Agricul-
ture Food Composition Databases (USDA). Current transversal study
comprised data on food intake of participants within the main research
theme 'Changes in Life Style and Cardiovascular Risks'. Data were an-
alyzed by Kruskal Wallis and Post-Hoc Dunn tests and by Pearson's
Co-relation test. Fifty-nine $24-\mathrm{h}$ records were evaluated. Medians of 21
out of 29 nutrients ( $72.4 \%$ ) showed statistically significant difference
(p $<0.05$ ) when compared to results from evaluated tables. There
was significant divergence between the nutrition rates in the lists. The
above is a warning on the use of these tools and their influence on re-
sults in calculating food intake and impact in nutritional prescriptions.

KEY WORDS: Food analysis; Food intake; Nutrients; List of Food Composition. 


\section{INTRODUÇÃO}

Os hábitos alimentares estão mergulhados em uma simbologia da vida social que envolve aspectos culturais, antropológicos, bem como experiências pessoais relacionadas a questões socioeconômicas, ambientais e psicológicas $^{1,2}$. Podem resultar em conflitos associados à preocupação com a saúde e aos valores de cada indivíduo. A vida moderna tem se mostrado desafiadora no que se refere às escolhas alimentares, especialmente nas cidades, ocorrendo uma modificação no estilo alimentar, migrando do ambiente doméstico para o ambiente comercial e gerando modificações na estrutura e rotina alimentares $^{3,4}$.

Diante destes desafios, investigar a prática alimentar de um grupo ou de indivíduos é tarefa complexa do profissional nutricionista ${ }^{5}$ e um procedimento necessário para um diagnóstico nutricional adequado ${ }^{2}$. Conhecer os padrões do consumo alimentar se constitui em um instrumento extremamente valioso de análise da relação entre o hábito alimentar e o estado de saúde da populaçã $0^{5,6}$; permite acompanhar o perfil alimentar e fornece subsídios para o desenvolvimento de políticas públicas direcionadas para grupos específicos da população $0^{7,8}$. Uma das tarefas mais complexas para um profissional da área da Nutrição é medir a quantidade de energia e de nutrientes consumida por um indivíduo, diariamente. Para a realização de uma avaliação segura e mais fidedigna possível, o profissional enfrenta dois pontos críticos: primeiro, obter, com exatidão, os dados referentes à ingestão alimentar e segundo, converter essas informações em valores quantitativos de energia e de nutrientes consumidos. Erros na avaliação do consumo alimentar podem estar relacionados a vieses, durante a coleta dos dados, e à metodologia usada para o cálculo da dieta. $\mathrm{O}$ cálculo da composição dos alimentos pode ser realizado através de softwares especialmente desenvolvidos para esta função. Outra forma de analisar a ingestão de nutrientes são as tabelas de composição de alimentos ou análise quími$\mathrm{Ca}^{9 \cdot 11}$. A obtenção destes dados de ingestão dietética é um processo complexo, pois a interpretação dos dados é diretamente influenciada pela quantidade de informações disponíveis na base de dados ${ }^{12,13}$.

Há algumas décadas, presencia-se uma lacuna com relação a pesquisas e publicações informativas sobre os nutrientes presentes nos alimentos brasileiros e, cada vez mais, tem se observado uma preocupação maior em relação a este assunto e uma cobrança no sentido de que se possa dispor de informações atualizadas e completas sobre a composição dos alimentos brasileiros. As tabelas de composição existentes, e até hoje utilizadas, não apresentam, em sua maioria, dados completos, não descrevem os processos empregados durante as análises, a composição e origem das receitas, o estado físico do alimento, se cru ou preparado e sua procedência. Assim, diferentes tabelas podem apresentar resultados discrepantes sobre o mesmo alimento ${ }^{14}$.

A par da importância da confiabilidade destes instrumentos, pilares na avaliação nutricional, no sentido de evitar resultados incorretos, que possam levar a orientações equivocadas, as tabelas precisam ser atualizadas e completas, baseadas em análises originais conduzidas de acordo com plano de amostragem representativo e métodos validados, a fim de fornecer informações que verdadeiramente representem a composição dos alimentos ${ }^{13}$.

Diante do exposto, o presente estudo objetivou comparar o resultado quantitativo do consumo alimentar, calculado a partir de duas tabelas de composição de alimentos, uma americana (United States Department of Agriculture - Food Composition Databases - USDA) ${ }^{11}$ e outra brasileira (Tabela Brasileira de Composição dos Alimentos - TACO $)^{10}$, complementada ou não por rótulos de alimentos, ambas comumente utilizadas na avaliação nutricional de indivíduos, tanto na prática clínica como em pesquisas epidemiológicas.

\section{METODOLOGIA}

Trata-se de um estudo transversal, descritivo analítico a partir de dados do consumo alimentar coletados no estudo principal denominado: Modificação do Estilo de Vida e Risco Cardiovascular (MERC) nos efeitos de diferentes intervenções de modificação do estilo de vida sobre aspectos físicos, metabólicos e comportamentais envolvidos na Síndrome Metabólica. O estudo principal foi conduzido no Centro de Reabilitação do Hospital São Lucas da Pontifícia Universidade Católica do Rio Grande do Sul (PUCRS), no período de setembro de 2010 a abril de 2013. 
Foram analisados cinquenta e nove recordatórios de $24 \mathrm{~h}$ (R24h) de participantes da pesquisa e que possuíam informações integralmente cadastradas na base de dados do estudo principal. O estudo incluiu homens e mulheres entre 30 e 59 anos, diagnosticados com Síndrome Metabólica segundo critérios da American Heart Association - National Hearth, Lung, and Blood Institu$t e^{15}$, ou seja, três ou mais dos seguintes achados: circunferência abdominal maior ou igual a $88 \mathrm{~cm}$ para mulheres e $102 \mathrm{~cm}$ para homens; pressão arterial sistólica acima de $130 \mathrm{mmHg}$ e diastólica, acima de $85 \mathrm{mmHg}$; glicose de jejum acima de $100 \mathrm{mg} / \mathrm{dl}$; triglicerídeos acima de 150 $\mathrm{mg} / \mathrm{dl}$; e HDL colesterol abaixo de $40 \mathrm{mg} / \mathrm{dl}$ para homens e abaixo de $50 \mathrm{mg} / \mathrm{dl}$ para mulheres ${ }^{15}$.

A composição nutricional dos R24h foi calculada de três formas: (1) utilizando-se o software brasileiro ADSNutri, alimentado com dados da $\mathrm{TACO}^{10}$, e para os alimentos não encontrados na TACO, foram utilizadas tabelas de medidas caseiras ${ }^{16,17}$ e informações contidas nos rótulos de alimentos como refrigerantes diet e $l i$ $g h t$, embutidos, pães, grãos integrais, leites e derivados, doces, bebidas alcoólicas, gorduras, biscoitos e algumas preparações, para equiparação com as encontradas na tabela americana; (2) utilizando-se o software brasileiro ADSNutri, alimentado com dados da TACO $^{10}$, exclusivamente; (3) utilizando-se o software americano Nutribase, alimentado com dados da United States Department of Agriculture - Food Composition Databases (USDA) ${ }^{11}$. As tabelas de composição químicas dos alimentos TACO e USDA foram escolhidas por serem amplamente utilizadas na prática da nutrição clínica e em pesquisas epidemiológicas e por possuírem exemplares disponíveis on-line.

As variáveis comparadas foram: consumo total de energia em quilocalorias, proteína em gramas, em quilocalorias e percentual de proteínas do valor energético total (VET); gordura total em gramas, em quilocalorias e percentual de gorduras do VET; frações de lipídeos (quantidade de gorduras saturadas, poliinsaturadas e monoinsaturadas); carboidratos em gramas, em quilocalorias e percentual de carboidratos do VET, quantidade de vitaminas A, B1, B2, B3 e dos minerais cálcio, magnésio, potássio, sódio, zinco e ferro. TRATAMENTO ESTATÍSTICO

Os dados foram armazenados em banco de dados Microsoft Excel e analisados por meio do Statistical Package for the Social Sciences (SPSS Inc., Chicago,
Ilinois, Estados Unidos) versão 21.0. A normalidade da distribuição dos dados foi verificada através do Teste Kolmogorov-Smirnof e todas as variáveis apresentaram distribuição não gaussiana. Os dados foram descritos por meio de medidas de tendência central e dispersão (mediana e intervalo interquartil) e a correlação entre os dados foi verificada através do Teste de Correlação de Spearman. A comparação entre as medidas resultantes das três formas de cálculo da composição nutricional foi realizada através do Teste de Kruskal Wallis e Post Hoc de Dunn. As análises foram realizadas considerando-se o nível de confiança de $95 \%(\alpha=5 \%)$.

\section{ASPECTOS ÉTICOS}

O estudo principal foi aprovado pelo Comitê de Ética da instituição, sob número 10/05153 e no Registro Brasileiro de Ensaios Clínicos, REBEC, sob número RBR-9 wz5fc e todos os participantes assinaram o Termo de Consentimento Livre e Esclarecido.

\section{RESULTADOS}

Foram avaliados 59 R24h de indivíduos diagnosticados com Síndrome Metabólica e as medianas de 21 entre 29 itens $(72,4 \%)$, que resultaram desta avaliação, apresentaram diferença estatisticamente significativa ( $p$ $<0,05)$ na comparação entre os resultados das três formas de cálculo da composição nutricional.

A USDA apresentou medianas superiores aos valores encontrados segundo a TACO exclusivamente, para 21 itens (VET total, carboidrato em gramas, gordura total em gramas, gordura total em kcal, gordura saturada, monoinsaturada, poliinsaturada, vitaminas A, B1, B2, B3, B9, B12, D, E, K, cálcio, magnésio, potássio, sódio, ferro).

Em relação à TACO $+\mathrm{R}$ as medianas da USDA foram superiores para 14 nutrientes (gordura monoinsaturada, vitaminas A, B1, B2, B3, B9, B12, D, E, K, magnésio, potássio, sódio e ferro).

$O$ VET e os percentuais de macronutrientes em relação ao VET, proteína (g e kcal), carboidrato (kcal), vitamina $\mathrm{C}$ e zinco não apresentaram diferença estatisticamente significativa a partir das distintas formas de cálculo de composição nutricional avaliadas (Tabela 1). 
Tabela 1. Comparação entre a quantidade de macro e de micronutrientes calculada a partir de três tabelas: USDA, TACO e TACO mais rótulos de alimentos $(\mathrm{N}=59)$

\begin{tabular}{|c|c|c|c|c|}
\hline Nutriente & $\begin{array}{l}\text { USDA } \\
\text { Mediana (II) }\end{array}$ & $\begin{array}{l}\text { TACO } \\
\text { Mediana (II) }\end{array}$ & $\begin{array}{l}\text { TACO } \\
+ \\
\text { Rótulos } \\
\text { Mediana (II) }\end{array}$ & $P$ \\
\hline VET (kcal) & $1758,0^{\mathrm{a}}(1356,0-2628,0)$ & $1425,7^{\mathrm{b}}(1055,8-1999,2)$ & $1789,9^{\mathrm{a}}(1308,5-2503,1)$ & 0,014 \\
\hline Proteína (g) & $78,0(61,0-122,0$ & $75,0(46,8-111,6)$ & $87,3(62,8-133,7)$ & 0,218 \\
\hline Proteína (kcal) & $328,0(246,0-506,0)$ & $299,9(187,4-446,5)$ & $349,3(251,0-534,8)$ & 0,179 \\
\hline Proteína (\% VET) & $19,0(15,0-23,0)$ & $21,2(14,6-27,4)$ & $20,0(15,0-24,5)$ & 0,302 \\
\hline Carboidrato (g) & $219,0^{\mathrm{a}}(151,0-327,0)$ & $174,6^{\mathrm{b}}(112,3-266,1)$ & $230,8^{\mathrm{a}}(145,9-319,2)$ & 0,033 \\
\hline Carboidrato (kcal) & $833,0(563,0-1220,0)$ & $698,4(449,1-1064,5)$ & $923,1(583,5-1276,6)$ & 0,058 \\
\hline Carboidrato (\% VET) & $45,0(38,0-56,0)$ & $49,9(39,6-63,0)$ & $47,2(41,3-59,9)$ & 0,458 \\
\hline Gordura total (g) & $61,0^{\mathrm{a}}(39,0-99,0)$ & $39,8^{\mathrm{b}}(26,1-61,2)$ & $50,7^{\mathrm{a}, \mathrm{b}}(33,5-80,4)$ & 0,013 \\
\hline Gordura total (kcal) & $555,0^{\mathrm{a}}(376,0-927,0)$ & $358,0^{\mathrm{b}}(235,3-551,1)$ & $456,2^{\mathrm{a}, \mathrm{b}}(301,2-723,2)$ & 0,007 \\
\hline Gordura total (\% VET) & $32,0(21,0-42,0)$ & $28,9(19,3-39,3)$ & $28,9(21,3-38,3)$ & 0,376 \\
\hline Gordura Saturada (g) & $24,0^{\mathrm{a}}(14,0-33,0)$ & $11,4^{b}(8,1-21,8)$ & $18,5^{\mathrm{a}, \mathrm{b}}(11,2-30,2)$ & 0,003 \\
\hline G. Monoinsaturada (g) & $21,0^{\mathrm{a}}(12,0-39,0)$ & $11,5^{\mathrm{b}}(7,9-21,3)$ & $14,5^{\mathrm{b}}(8,8-22,3)$ & 0,002 \\
\hline G. Poliinsaturada (g) & $9,0^{\mathrm{a}}(5,0-16,0)$ & $5,2^{\mathrm{b}}(2,8-10,4)$ & $6,1^{\mathrm{a}, \mathrm{b}}(3,1-11,7)$ & 0,003 \\
\hline Vitamina A $(\mu \mathrm{g})$ & $568,0^{\mathrm{a}}(284,0-855,0)$ & $58,9^{\mathrm{b}}(9,1-154,9)$ & $126,7^{\mathrm{b}}(28,2-304,3)$ & $<0,001$ \\
\hline Vitamina B1 (mg) & $1,0^{\mathrm{a}}(1,0-2,0)$ & $0,8^{\mathrm{b}}(0,5-1,1)$ & $0,8^{\mathrm{b}}(0,5-1,3)$ & $<0,001$ \\
\hline Vitamina B2 (mg) & $2,0^{\mathrm{a}}(1,0-2,0)$ & $0,8^{\mathrm{b}}(0,3-1,3)$ & $0,8^{\mathrm{b}}(0,3-1,3)$ & $<0,001$ \\
\hline Vitamina B3 (mg) & $20,0^{\mathrm{a}}(13,0-31,0)$ & $14,7^{\mathrm{b}}(6,3-26,7)$ & $14,7^{\mathrm{b}}(6,4-27,0)$ & 0,015 \\
\hline Vitamina B9 (mg) & $94,0^{\mathrm{a}}(37,0-167,0)$ & $0,0^{\mathrm{b}}(0,0-0,0)$ & $0,0^{\mathrm{b}}(0,0-0,0)$ & $<0,001$ \\
\hline Vitamina B12 ( $\mu \mathrm{g})$ & $5,0^{\mathrm{a}}(3,0-9,0)$ & $0,0^{\mathrm{b}}(0,0-0,0)$ & $0,0^{\mathrm{b}}(0,0-0,0)$ & $<0,001$ \\
\hline Vitamina C (mg) & $130,0(47,0-227,0)$ & $75,0(19,4-206,7)$ & $75,0(22,3-213,0)$ & 0,127 \\
\hline Vitamina D (UI) & $59,0^{a}(24,0-138,0)$ & $0,0^{\mathrm{b}}(0,0-0,0)$ & $0,0^{\mathrm{b}}(0,0-0,0)$ & $<0,001$ \\
\hline Vitamina E (UI) & $6,0^{\mathrm{a}}(2,0-9,0)$ & $0,0^{\mathrm{b}}(0,0-0,0)$ & $0,0^{\mathrm{b}}(0,0-0,0)$ & $<0,001$ \\
\hline Vitamina K ( $\mu \mathrm{g})$ & $53,0^{\mathrm{a}}(28,0-137,0)$ & $0,0^{\mathrm{b}}(0,0-0,0)$ & $0,0^{\mathrm{b}}(0,0-0,0)$ & $<0,001$ \\
\hline Cálcio (mg) & $675,0^{\mathrm{a}}(463,0-915,0)$ & $483,5^{\mathrm{b}}(236,3-782,6)$ & $527,7^{\mathrm{a}, \mathrm{b}}(378,0-861,2)$ & 0,011 \\
\hline Magnésio (mg) & $310,0^{a}(199,0-415,0)$ & $197,6^{\mathrm{b}}(132,1-288,5)$ & $197,6^{\mathrm{b}}(132,1-288,5)$ & $<0,001$ \\
\hline Potássio (mg) & $2777,0^{\mathrm{a}}(1954,0-3794,0)$ & $1988,6^{\mathrm{b}}(1436,0-2881,0)$ & $2131,3^{\mathrm{b}}(1592,7-2881,0)$ & 0,001 \\
\hline Sódio (mg) & $2078,0^{\mathrm{a}}(1656,0-3623,0)$ & $1155,1^{c}(610,5-1838,5)$ & $1612,0^{\mathrm{b}}(1080,4-2497,0)$ & $<0,001$ \\
\hline Zinco (mg) & $10,0(7,0-16,0)$ & $8,0(5,7-13,2)$ & $9,1(5,7-14,6)$ & 0,183 \\
\hline Ferro (mg) & $12,0^{\mathrm{a}}(9,0-17,0)$ & $7,3^{\mathrm{b}}(4,6-10,8)$ & $8,7^{\mathrm{b}}(5,3-12,6)$ & $<0,001$ \\
\hline
\end{tabular}

Teste Kruskal Wallis e Post Hoc de Dunn; II: Intervalo interquartil; VET: Valor Energético Total; G: gordura. USDA: Food Composition Databases; TACO: Tabela Brasileira de Composição dos Alimentos. 
Avaliando a correlação entre USDA e TACO (Tabela 2), observou-se que 11 nutrientes apresentaram correlação positiva forte $(s=0,6$ a 0,$9 ; \mathrm{p}<0,001)$, e 13, correlação positiva regular ( $s=0,3$ a 0,$5 ; \mathrm{p}<0,01)$. As vitaminas $\mathrm{B} 12$, D e E não apresentaram correlação estatisticamente significativa.

Tabela 2. Correlação entre a quantidade de macro e de micronutrientes calculada a partir da USDA versus a TACO $(\mathrm{N}=59)$

\begin{tabular}{|c|c|c|}
\hline Nutriente & $s$ & $P$ \\
\hline VET (kcal) & 0,676 & $<0,001$ \\
\hline Proteína (g) & 0,753 & $<0,001$ \\
\hline Proteína (kcal) & 0,753 & $<0,001$ \\
\hline Proteína (\% VET) & 0,558 & $<0,001$ \\
\hline Carboidrato (g) & 0,620 & $<0,001$ \\
\hline Carboidrato (kcal) & 0,661 & $<0,001$ \\
\hline Carboidrato (\% VET) & 0,595 & $<0,001$ \\
\hline Gordura total (g) & 0,676 & $<0,001$ \\
\hline Gordura total (kcal) & 0,682 & $<0,001$ \\
\hline Gordura total (\% VET) & 0,598 & $<0,001$ \\
\hline Gordura Saturada (g) & 0,642 & $<0,001$ \\
\hline Gordura Monoinsaturada (g) & 0,672 & $<0,001$ \\
\hline Gordura Poliinsaturada (g) & 0,466 & $<0,001$ \\
\hline Vitamina A $(\mu \mathrm{g})$ & 0,343 & 0,008 \\
\hline Vitamina B1 (mg) & 0,558 & $<0,001$ \\
\hline Vitamina B2 (mg) & 0,421 & 0,001 \\
\hline Vitamina B3 (mg) & 0,359 & 0,005 \\
\hline Vitamina B9 (mg) & $\ddagger$ & $\ddagger$ \\
\hline Vitamina B12 ( $\mu \mathrm{g})$ & $-0,060$ & 0,654 \\
\hline Vitamina C (mg) & 0,592 & $<0,001$ \\
\hline Vitamina D (UI) & 0,010 & 0,942 \\
\hline Vitamina E (UI) & 0,102 & 0,444 \\
\hline Vitamina $\mathrm{K}(\mu \mathrm{g})$ & $\ddagger$ & $\ddagger$ \\
\hline Cálcio (mg) & 0,533 & $<0,001$ \\
\hline Magnésio (mg) & 0,694 & $<0,001$ \\
\hline Potássio (mg) & 0,555 & $<0,001$ \\
\hline Sódio (mg) & 0,393 & 0,002 \\
\hline Zinco (mg) & 0,595 & $<0,001$ \\
\hline Ferro (mg) & 0,654 & $<0,001$ \\
\hline
\end{tabular}

Correlacionando USDA com TACO $+\mathrm{R}$ (Tabela 3), a maioria dos nutrientes (19) apresentou correlação positiva forte $(s=0,6$ a 0,$9 ; \mathrm{P}<0,001)$ (VET, proteína, carboidrato, gordura total, saturada, monoinsaturada, vitamina $\mathrm{C}$ e todos os minerais. Gordura poliinsaturada e vitaminas B1, B2, B3 apresentaram correlação positiva regular $(s=0,3$ a 0,$5 ; \mathrm{P}<0,002)$ e vitamina $\mathrm{A}$, correlação positiva fraca $(s=0,296 ; \mathrm{P}=0,023)$. As vitaminas $\mathrm{B} 9, \mathrm{~B} 12$, $\mathrm{D}$, E e K não apresentaram correlação estatisticamente significativa.

Tabela 3. Correlação entre a quantidade de macro e de micronutrientes calculada a partir da USDA versus a TACO mais rótulos de alimentos $(\mathrm{N}=59)$

\begin{tabular}{|c|c|c|}
\hline Nutriente & $s$ & $P$ \\
\hline VET (kcal) & 0,831 & $<0,001$ \\
\hline Proteína (g) & 0,826 & $<0,001$ \\
\hline Proteína (kcal) & 0,822 & $<0,001$ \\
\hline Proteína (\% VET) & 0,623 & $<0,001$ \\
\hline Carboidrato (g) & 0,757 & $<0,001$ \\
\hline Carboidrato (kcal) & 0,797 & $<0,001$ \\
\hline Carboidrato (\% VET) & 0,704 & $<0,001$ \\
\hline Gordura total (g) & 0,837 & $<0,001$ \\
\hline Gordura total (kcal) & 0,841 & $<0,001$ \\
\hline Gordura total (\% VET) & 0,732 & $<0,001$ \\
\hline Gordura Saturada (g) & 0,872 & $<0,001$ \\
\hline Gordura Monoinsaturada (g) & 0,692 & $<0,001$ \\
\hline Gordura Poliinsaturada (g) & 0,503 & $<0,001$ \\
\hline Vitamina A ( $\mu \mathrm{g})$ & 0,296 & 0,023 \\
\hline Vitamina B1 (mg) & 0,559 & $<0,001$ \\
\hline Vitamina B2 (mg) & 0,417 & 0,001 \\
\hline Vitamina B3 (mg) & 0,399 & 0,002 \\
\hline Vitamina B9 (mg) & $-0,116$ & 0,383 \\
\hline Vitamina B12 ( $\mu \mathrm{g})$ & $-0,060$ & 0,654 \\
\hline Vitamina C (mg) & 0,639 & $<0,001$ \\
\hline Vitamina D (UI) & 0,010 & 0,942 \\
\hline Vitamina E (UI) & 0,102 & 0,444 \\
\hline Vitamina K ( $\mu \mathrm{g})$ & $\ddagger$ & $\ddagger$ \\
\hline Cálcio (mg) & 0,679 & $<0,001$ \\
\hline Magnésio (mg) & 0,695 & $<0,001$ \\
\hline Potássio (mg) & 0,635 & $<0,001$ \\
\hline Sódio (mg) & 0,691 & $<0,001$ \\
\hline Zinco (mg) & 0,639 & $<0,001$ \\
\hline Ferro (mg) & 0,614 & $<0,001$ \\
\hline
\end{tabular}

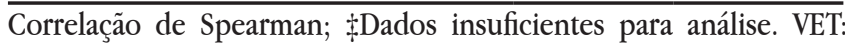
Valor Energético Total; USDA: Food Composition Databases; TACO: Tabela Brasileira de Composição dos Alimentos. 


\section{DISCUSSÃO}

O objetivo deste estudo foi comparar o consumo alimentar calculado a partir de duas tabelas de composição dos alimentos e os resultados demonstraram divergência em valores encontrados a partir do cálculo do R24h pela tabela americana USDA e pela tabela brasileira TACO, tanto na versão em que foram considerados rótulos de alimentos como na versão sem considerar rótulos de alimentos. A maioria dos itens analisados apresentou diferença na comparação entre os resultados, entretanto, a diferença entre a USDA e a TACO foi mais significativa que a diferença entre a USDA e a TACO + R. Pode-se observar, também, que, em geral, as quantidades foram menores na TACO, em relação à USDA, sugerindo que a USDA seja mais abrangente e completa pois alguns nutrientes não são avaliados na TACO.

Somente as quantidades relativas a oito nutrientes não apresentaram diferença, quando consideradas as três tabelas, entre eles, os valores relativos a proteína. A tabela TACO foi desenvolvida em fases, iniciadas em 1996, e em sua fase II, de 1999 a 2001, foram identificadas as marcas de alimentos mais consumidas e estas foram levadas para análise. Entre as proteínas, foram adquiridas carnes bovinas, aves e pescados e realizadas as análises laboratoriais. Algumas características das fontes proteicas alimentares consumidas pela população em geral podem contribuir para que o resultado da análise destes nutrientes traga informações similares: a existência de um número reduzido de tipos de carnes, ou seja, bovinas, suínas, aves e pescados. Exceto pelos pescados, que englobam um número maior de espécies, as demais fontes proteicas são originárias de poucas espécies. Por outro lado, são produtos analisados in natura e que sofrem poucas alterações relacionadas ao processo industrial ou à adição de outros componentes. Estes aspectos poderiam explicar o fato de produtos proteicos não terem apresentado diferença significativa entre as tabelas analisadas, mesmo considerando as diferenças decorrentes da região de criação e da alimentação fornecida aos animais em análise.

A não observância de diferença nas quantidades de vitamina $\mathrm{C}$ pode estar associada ao fato de vegetais, em geral, serem boas fontes de vitamina $\mathrm{C}^{18,19} \mathrm{e}$ de os níveis de vitamina $\mathrm{C}$ serem reportados como a soma dos ácidos ascórbico e desidroascórbico nas análises laboratoriais ${ }^{1,20}$, na maioria dos países, tornando estes dados comparáveis mesmo a despeito dos métodos analíticos utilizados $^{10,14,21}$.

A tabela USDA é utilizada para a estimativa de nutrientes em todo o mundo, muitas vezes sem que sejam consideradas as diferenças que existem na composição nutricional. Por outro lado, em um mundo globalizado, com mercado internacional onde são importados e exportados alimentos, o conteúdo de nutrientes das tabelas de composição nutricional tende a apresentar uma abordagem cada vez mais global ${ }^{22}$.

A forte correlação entre a maioria das variáveis analisadas demonstra um comportamento similar entre elas, fato positivo. Mas para algumas vitaminas não houve correlação (B9, B12, D, E) e uma das razões pode ser atribuída ao fato de a TACO não disponibilizar informações sobre estas vitaminas.

Entretanto, as medianas de alguns nutrientes apresentaram diferenças bastante elevadas, especialmente os micronutrientes. Na literatura não há informações disponíveis que possam justificar as diferenças encontradas. As razões podem ser as mais diversas, desde aquelas relacionadas ao alimento em si, suas características intrínsecas e extrínsecas, como os diferentes métodos de análise utilizados para identificação da sua composição e o fato, também, de as tabelas nutricionais não disponibilizarem informações completas. Alguns micronutrientes não são avaliados, como vitaminas B9, B12, D, K e E, que não são disponibilizadas pela TACO.

As medianas dos VETs calculados segundo a USDA e a TACO foram significativamente diferentes. Entretanto, quando complementada a TACO com rótulos dos alimentos não presentes na tabela, a comparação entre os VETs não mais apresentou diferença, indicando a necessidade de se complementar a avaliação do consumo alimentar com informações de alimentos não presentes na tabela.

Cada tabela de composição de alimentos é gerada a partir da análise dos alimentos de determinada região, com suas características específicas e através de determinados métodos. Assim, a diferença encontrada na maioria dos nutrientes demonstra que o uso de tabelas de composição de alimentos estruturadas em outros 
países, como é o caso da USDA, pode influenciar na avaliação nutricional de uma dieta, sendo assim, o uso de tabelas que não foram constituídas a partir da análise dos alimentos locais pode apresentar limitações e comprometer a avaliação do consumo alimentar ${ }^{23-25}$.

As diferentes formas de análises utilizadas pelas tabelas podem acarretar uma diferenciação dos valores de nutrientes encontrados. Variações ambientais, como solo, clima, variedade genética (cultivares), processamento e armazenamento também podem contribuir, acarretando uma dificuldade no parâmetro avaliativo, principalmente quando se trata de vegetais e seus derivados ${ }^{12,26}$.

Os dados obtidos pelo presente estudo evidenciam a importância de conhecer a origem e estruturação das tabelas de composição dos alimentos, que servem não somente como uma ferramenta básica para os profissionais de nutrição, mas para outros setores de saúde.

Outros estudos comparando tabelas de composição de alimentos não estão disponíveis; entretanto, existem pesquisas que realizaram a comparação de softwares. Lourenço et al. ${ }^{27}$ compararam três softwares, evidenciando que não havia diferença estatística significativa com relação a energia, macronutrientes e micronutrientes; no entanto, detectaram divergência entre as gramaturas para medidas caseiras encontradas em cada software. Por outro lado, Vieira et $\mathrm{al}^{28}$, ao compararem o valor nutricional de dez cardápios, através de quatro softwares, também não encontraram diferença estatística significativa em energia e macronutrientes, porém, em dois dos programas utilizados, verificaram diferença de valores em frações lipídicas, ferro e sódio.

Por sua vez, Ribeiro et al. ${ }^{12}$ citaram que a análise laboratorial seria a forma mais adequada de se realizar a avaliação do consumo alimentar de indivíduos. No entanto, é inviável que o profissional nutricionista na sua prática clínica realize esta análise laboratorial do consumo alimentar de seus pacientes, visto o alto custo dessa prática e o tempo necessário para esta análise; sendo assim, o uso das tabelas de composição dos alimentos se torna $\mathrm{o}$ instrumento recomendado e mais utilizado.

A análise do consumo alimentar através do R24h apresenta, por sua vez, limitações, pois apesar de amplamente utilizado por ser de fácil aplicação, a qualidade da informação coletada depende da memória e da cooperação do paciente, além de estimar valores absolutos ou relativos à ingestão de nutrientes. Durante a realização do estudo, houve dificuldade na realização da análise de dados do grupo de pesquisa do estudo principal, denominado Modificação do Estilo de Vida e Risco Cardiovascular (MERC), devido à imprecisão das medidas encontradas e à dificuldade de mensuração das medidas caseiras, pelos participantes ${ }^{29}$.

Considerando a importância de dispor de dados mais completos sobre as tabelas de composição dos alimentos, salienta-se a contribuição deste trabalho como fonte de dados para futuras pesquisas condizentes com a realidade dos hábitos alimentares nos dias de hoje.

Diante de dois ramos de pensamento muito antigos sobre tabelas de composição de alimentos, e que se perpetuam até hoje, temos um, que tende a considerar que os números nelas contidos devem ter a precisão do peso atômico e outro, que pretende destituir seu valor alegando que o alimento sofre modificações pelo solo, época de cultivo, taxa de crescimento e que não é confiável para cálculo da composição de uma dieta. A verdade deve estar em algum lugar entre estes dois pontos de vista $^{30}$.

Diante do desenvolvimento e da mudança de hábitos alimentares da população, faz-se necessário que as tabelas de composição química dos alimentos sejam constantemente atualizadas, complementadas e revisadas a fim de fornecerem informações mais precisas, que acompanhem o crescimento e desenvolvimento globais, com novos alimentos, novos hábitos e novos métodos de análise. Enquanto não houver um sistema adequado e atual, é necessário que os nutricionistas sejam realistas em relação à acurácia das informações contidas nas tabelas de composição de alimentos.

\section{CONCLUSÃO}

Foram encontradas divergências significativas entre os valores nutricionais apresentados nas tabelas $\mathrm{da}$ USDA e TACO. Este fato alerta para o uso destes instrumentos e a sua influência nos resultados do cálculo do consumo alimentar e da prescrição nutricional. Ressalta a necessidade de se complementarem as informações com dados de rótulos de alimentos não constantes nas tabelas de composição. 


\section{AGRADECIMENTOS}

Ao Conselho Nacional de Desenvolvimento Científico e Tecnológico (CNPq) e à Fundação de Amparo à Pesquisa do Estado do Rio Grande do Sul (FAPERGS), fontes de financiamento da pesquisa.

\section{REFERÊNCIAS}

1. Stajcic N. Undestanding Culture-food as a means of communication. Hemispheres. 2015; 28:5-14.

2. Moraes RW. Determinantes e construção do comportamento alimentar, uma revisão narrativa da literatura. [dissertação]. Porto Alegre (RS): Universidade Federal do Rio Grande do Sul; 2014.

3. Garcia RWD. Representações sobre consumo alimentar e suas implicações em inquéritos alimentares: estudo qualitativo em sujeitos submetidos à prescrição dietética. Rev Nutr. 2004; 17(1):15-28.

4. Louzada M, Martins A, Canella D, Baraldi L, Levy R, VClaro R, et al. Alimentos ultraprocessados e perfil nutricional da dieta no Brasil. Rev Saude Publica. 2015:49-38.

5. CFN Conselho Federal de Nutricionistas. Resolução CFN 417 - Dispõe sobre procedimentos nutricionais para atuação dos nutricionistas e dá outras providências. Brasília; 2008 [acesso em 2018 mar. 27]. Disponível em: http://www.cfn.org.br/novosite/pdf/ res/2008/res417.pdf.

6. Elmadfa I, Meyer AL. Importance of food composition data to nutrition and public health. Eur J Clin Nutr. 2010; 64(Suppl 3):S4-7.

7. Brasil. Guia alimentar para a população brasileira. Ministério da Saúde, Secretaria de Atenção à Saúde, Departamento de Atenção Básica; 2014.

8. Gomes AA, Pereira RAP, Yokoo EM. Caracterização do consumo alimentar de adultos por meio de questionário simplificado: contribuição para os estudos de vigilância alimentar e nutricional. Cad Saude Coletiva. $2015 ; 23(4): 368-73$.

9. Finglas $\mathrm{P}$, Roe M, Pinchen H, Astley S. The contribu- tion of food composition resources to nutrition science methodology. Nutrition Bull. 2017.

10. Tabela Brasileira de Composição de Alimentos/NEPA - UNICAMP. 4 ed. rev. e ampl. Campinas: NEPA-UNICAMP, 2011. 161p.

11. USDA. Food Composition Databases: United States Department of Agriculture. Agricultural Research Service; 2017. Disponível em: https://ndb.nal.usda. gov/ndb/.

12. Ribeiro P, Morais TB, Colugnati FAB, Sigulem DM. Tabelas de composição química de alimentos: análise comparativa com resultados laboratoriais. Rev Saude Publica. 2003; 37(2):216-25.

13. Marconi S, Durazzo A, Camilli E, Lisciani S, Gabrielli P, Aguzzi A, et al. Food Composition Databases: Considerations about Complex Food Matrices. Foods. 2018; 7(1):2.

14. Drehmer M, Melere C, Reinheimer SM, Camey SA. Agreement of dietary fiber and calorie intake values according to the choice of nutrient composition and household measure tables. Rev Nutr. 2017;30:23344 .

15. Grundy SM, Cleeman JI, Daniels SR, Donato KA, Eckel RH, Franklin BA, et al. Diagnosis and management of the metabolic syndrome: an American Heart Association/National Heart, Lung, and Blood Institute Scientific Statement. Circulation. 2005; 112 (17):2735-52.

16. Monego E, Peixoto M, Santiago R, Gil M, Cordeiro M, Campos M, et al. Alimentos brasileiros e suas porções: um guia para avaliação do consumo alimentar. Rio de Janeiro: Rubio; 2013.

17. Pinheiro ABV, Lacerda EMA, Benzecry EH, Gomes MCS, Costa VM. Tabela para Avaliação de Consumo Alimentar em Medidas Caseiras. São Paulo: Atheneu; 2005.

18. Carr AC, Vissers MCM. Synthetic or Food-Derived Vitamin C - Are They Equally Bioavailable? Nutrients. 2013; 5(11):4284-304.

19. Hackbarth RDT, Thieme RD, Schieferdecker MEM. Vitamina C - Ácido Ascórbico. In: Schieferdecker MEM, 
Thieme RD, Haushild DB, editors. Vitaminas, Minerais e Eletrólitos. Rio de Janeiro: Rubio; 2016. 344p.

20. Behrens WA, Madere R. Quantitative Analysis of Ascorbic Acid and Isoascorbic Acid in Foods by High-Performance Liquid Chromatography with Electrochemical Detection. J Liq Chromatogr. 1994;17:2445-55.

21. Deharveng G, Charrondière UR, Climani N, Southgate DAT, Riboli E. Comparison of nutrients in the food composition tables available in the nine European countries participating in EPIC. Eur J Clin Nutr. 1999;53:60-79.

22. Kearney J. Food consumption trends and drivers. Philos Trans R Soc Lond B Biol Sci. 2010; 365(1554): 2793-807.

23. Konstansa L, Dusica S, Dragan B. Estimation of Macronutrient Content in Kindergartens Meals: Food Composition Tables or Chemical Analysis? Iran J Pediatr. 2014; 24(5):643-6.

24. Bruyn J, Ferguson E, Allman-Farinelli M, Darnton-Hill I, Maulaga W, Msuya J, et al. Food composition tables in resource-poor settings: exploring current limitations and opportunities, with a focus on animal-source foods in sub-Saharan Africa. Br J Nutr. 2016; 116(10):1709-19.

25. Chege PM, Ndungu ZW. Opportunities and Limitations of Using Food Composition Tables in Clinical Nutrition and Dietetics in Kenya. E-Cronicon. 2016: 24-7.

26. Martín ISM, Rodríguez BH. Herramientas para la calibración de menús y cálculo de la composición nutricional de los alimentos; validez y variabilidad. Nutr Hosp. 2014; 29(4):929-34.

27. Lourenço PKAC, Castro JL, Vale SHL, Alves CX, Leite LD. Comparação de três programas computacionais utilizados na avaliação de recordatórios alimentares 24 horas. J Health Inform. 2011;3(1):13-8.

28. Vieira FGK, Di Pietro PF, Feio LC, Assis MAA, Peres MA, Vasconcelos FAG. Comparação do valor nutricional de dez cardápios segundo quatro programas computacionais. Rev Nutr. 2009; 22(1):29-38. tos Alimentares na Avaliação do Consumo alimentar. Rev Brasil Ciencias Saude. 2015;19(2):91-100.

30. Widdowson EM, McCance RA. Food tables, their scope and limitations. Lancet. 1943;1:230-2.

Recebido em: 24/07/2018

Aceito em: 18/09/2018



